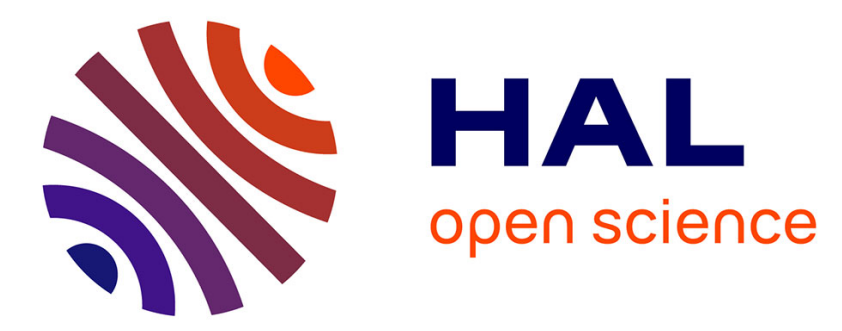

\title{
Nicolas Marquis, Du bien-être au marché du malaise. La société du développement personnel
}

Maël Dieudonné

\section{To cite this version:}

Maël Dieudonné. Nicolas Marquis, Du bien-être au marché du malaise. La société du développement personnel. 2014, http://lectures.revues.org/16223. halshs-01094166

\section{HAL Id: halshs-01094166 \\ https://shs.hal.science/halshs-01094166}

Submitted on 15 Dec 2014

HAL is a multi-disciplinary open access archive for the deposit and dissemination of scientific research documents, whether they are published or not. The documents may come from teaching and research institutions in France or abroad, or from public or private research centers.
L'archive ouverte pluridisciplinaire HAL, est destinée au dépôt et à la diffusion de documents scientifiques de niveau recherche, publiés ou non, émanant des établissements d'enseignement et de recherche français ou étrangers, des laboratoires publics ou privés. 
Nicolas Marquis, Du bien-être au marché du
malaise. La société du développement personnel

Maël Dieudonné

Doctorant en sociologie à l'université Lyon 2, rattaché au Centre Max Weber

$20 / 11 / 2014$

À travers ce livre ${ }^{1}$, Nicolas Marquis propose d'analyser une pratique sociale traditionnellement décriée mais assurément peu comprise : la lecture d'ouvrages de « développement personnel» (DP). Ce genre littéraire se distingue par la possibilité qu'il offre à ses lecteurs de «travailler sur eux-mêmes » afin de maitriser certaines dimensions concrètes de leur existence (émotions, comportements, santé). Il connaît actuellement un vif succès, difficile à quantifier mais incitant à y reconnaître le révélateur de faits sociaux. Pour l'analyser, l'auteur s'appuie sur un matériau empirique abondant, collecté par ses soins. Il consiste d'une part en 55 entretiens compréhensifs avec des lecteurs de DP, recrutés à l'aide de petites annonces placées dans quatre grandes librairies de la Belgique francophone, d'autre part en 295 lettres adressées à trois auteurs de DP : Boris Cyrulnik, Thomas d'Ansembourg et Thierry Janssen (avec lesquels ont également été réalisés des entretiens au sujet des courriers qu'ils reçoivent habituellement). Le propos est découpé en sept chapitres.

Dans le premier chapitre, sont présentées les analyses traditionnelles du DP, qui s'avèrent fortement critiques. Celles relevant $d u$ "modèle du déclin » l'érigent en symptôme d'un malaise culturel, au motif qu'il invite les individus à se désinvestir de la sphère publique et des rapports sociaux, pour se concentrer sur leur jouissance personnelle et sur des expériences de subjectivation; au contraire, les analyses s'inscrivant dans le «modèle du pouvoir » reconnaissent en le DP un instrument de domination, pour la raison qu'il contraint les individus à se concevoir comme responsables de phénomènes relevant plutôt de logiques sociales (il impose notamment des stéréotypes de genre). Ces analyses supposent toutes que les ouvrages de DP agissent sur leurs lecteurs, dont ils font des sujets gouvernables. Mais elles ne précisent jamais dans quelle mesure ni comment: pour Nicolas Marquis, ce n'est pas la moindre de leurs faiblesses.

L'auteur se consacre alors, dans le second chapitre, à l'élaboration d'un modèle d'analyse du DP dans lequel ses lecteurs ne soient pas «transparents ». Il s'appuie sur une présentation des théories de la réception, dont les auteurs se sont précisément attachés à expliquer comment un texte peut exercer des effets sur ses lecteurs, et ont mis en évidence une double nécessité : celle de tenir compte des intentions des lecteurs, dont la visée est parfois plus utilitaire qu'esthétique; celle d'analyser le travail d'actualisation des textes auquel ils se livrent. Il conclut en proposant d'analyser la lecture d'ouvrages de DP comme une interaction entre un dispositif et des acteurs « disposés », produisant une expérience particulière.

\footnotetext{
${ }^{1}$ Issu d'une thèse de doctorat en sociologie soutenue en 2012 et récompensée par le Prix Le Monde de la recherche universitaire.
} 
Il s'agit ensuite de caractériser ce dispositif que constituent les textes de DP : c'est l'objet du troisième chapitre. Nicolas Marquis l'envisage d'abord dans sa dimension idéologique, c'est-à-dire selon les représentations qui y affleurent de façon récurrente. Il en identifie dix, qui convergent pour inviter les individus à se rapprocher de ce qui provient de leur intériorité (expression de leur nature authentique et fondamentalement positive), et à s'éloigner de ce qui provient de leur extériorité (émotions négatives, mauvaises pensées, etc.). Puis il étudie le dispositif formé par les ouvrages de DP dans sa dimension pratique, c'est-à-dire selon les sollicitations que ceux-ci adressent à leurs lecteurs. Deux apparaissent nettement : implicitement, les livres de de DP enjoignent leurs lecteurs à reconnaître la capacité du texte à changer effectivement leur situation personnelle (" connivence passive ») et ils sollicitent de leur part un effort conscient pour le mettre en relation avec elle ( " coopération active »).

$\mathrm{Au}$ chapitre suivant, sont explicitées les raisons que peuvent avoir des individus de répondre favorablement à ces sollicitations, c'est-à-dire leur « disposition». La lecture d'ouvrages de DP s'avère prendre sens dans un contexte biographique particulier - en l'occurrence, elle succède à l'ouverture d'une «brèche » dans leur vie quotidienne. Confrontés à une situation (quelles que soient sa nature et sa gravité objectives) générant un malaise suffisant pour les amener au constat que les choses doivent changer, échouant par ailleurs à trouver de l'aide dans les supports traditionnels, les individus procèdent à une «double ouverture » : ils acceptent de soumettre à révision certains aspects de leur existence (hygiène mentale, comportements face à autrui... ce qui implique de reconnaître qu'ils sont partiellement responsables de leur problème), et ils recherchent une assistance auprès de supports inhabituels, comme les ouvrages de DP. C'est donc avec une forte attente d'efficacité qu'ils consultent ces derniers.

Dans le chapitre 5, sont précisées les conditions auxquelles les individus acceptent de faire confiance aux livres de DP. Ces ouvrages doivent être engageants, c'est-à-dire tenir des propos d'apparence raisonnable, et s'adresser à leurs lecteurs comme à des personnes responsables et autonomes. Ils doivent également être crédibles, c'est-à-dire s'appuyer sur des preuves, dont les plus convaincantes ne sont pas scientifiques : elles relèvent plutôt de l'expérience personnelle des auteurs, qui généralement ont eux-mêmes surmonté une brèche, ou aidé d'autres personnes à le faire. Pour les lecteurs en effet, il s'agit moins d'évaluer la véracité des ouvrages de DP que leur plausibilité : peuvent-ils raisonnablement croire à leur efficacité ? Lorsqu'ils en sont convaincus, ils ne s'ouvrent pas seulement à la possibilité que leurs contenus les concernent personnellement, mais ils travaillent encore à la concrétiser, en s'efforçant d'appliquer le propos du livre à leur situation. Cette dernière perd alors de sa fatalité, puisqu'elle s'avère avoir déjà été surmontée par les personnes citées en exemple dans les ouvrages, à l'aide des conseils qu'ils fournissent.

Nicolas Marquis interroge au chapitre 6 les conditions de possibilité de ces « expériences de connexion » qui surviennent lorsque des individus se reconnaissent dans un ouvrage : il les repère dans les affinités culturelles et symboliques existant entre les ouvrages de DP et leurs lecteurs. L'idéologie des premiers trouve en effet 
de puissants échos dans les représentations des seconds, qui puisent leur sens dans les mêmes présupposés - le plus fondamental étant « de traduire une déconvenue dans les termes d'une pratique insuffisante de son intériorité » (p. 173). Cette complicité explique pourquoi les enquêtés ne contestent jamais le bien fondé du DP comme genre littéraire, alors qu'ils savent se montrer très critiques vis-à-vis d'ouvrages particuliers.

Arrivé au terme de l'analyse de la pratique consistant à lire des ouvrages de DP, l'auteur entreprend dans un dernier chapitre d'élucider sa signification sociale : que révèle cette pratique de la société dans laquelle elle prend place ? Malgré son apparente nouveauté, elle exprime une attitude très ancienne face à la contingence, assimilée par les anthropologues classiques à la pensée magique. Les raisonnements magiques comme ceux des lecteurs de DP visent à expliquer non seulement le malheur, mais surtout les circonstances dans lesquelles il frappe. Ils permettent chacun de répondre à deux questions : pourquoi cela arrive-t-il à moi (plutôt qu'à un autre) ? Pourquoi cela survient-il ici et maintenant (plutôt qu'ailleurs et dans un autre temps) ? Ces raisonnements confèrent ainsi une signification au malheur, en l'inscrivant dans un système d'imputation - l'originalité du DP étant de l'attribuer à ses victimes plutôt qu'à des personnes ou à des entités malveillantes. Ils permettent également d'agir sur le malheur, c'est-à-dire d'en atténuer la fatalité : le raisonnement magique, parce qu'il est historiquement lié à des procédures rétributives ; le raisonnement des lecteurs de DP, parce qu'il ouvre la possibilité de travailler sur soi. Nicolas Marquis conclut en avançant l'hypothèse que cette « forme de pensée magique à la moderne » (p. 189) constitue une manière de gérer les tensions provoquées par les injonctions à vivre librement, qui circulent aujourd'hui dans la société, chez les individus ne disposant pas de ressources suffisantes pour les réaliser.

Du bien-être au marché du malaise est un ouvrage plaisant à lire. Ses qualités littéraires sont d'abord indéniables : il est écrit dans un style clair et accessible, propose une démonstration explicite et progressive, et s'appuie sur de nombreux extraits d'entretiens et de courriers des lecteurs qui donnent une solide assise empirique au propos. Les pratiques et les représentations des lecteurs de DP sont analysées très finement, suscitant une réelle impression de comprendre ces derniers. L'ensemble constitue une belle illustration de ce que la sociologie gagne à considérer sérieusement des objets illégitimes. Malheureusement, le raisonnement souffre à notre avis d'une importante faiblesse, qui est d'ignorer le caractère potentiellement situé de la pratique étudiée. Au chapitre 6 par exemple, la possibilité que les représentations observées chez les personnes enquêtées entretiennent un rapport d'homologie avec certaines positions sociales n'est jamais évoquée. Les lecteurs d'ouvrages de DP sont seulement caractérisés par le fait d'avoir été affectés par une brèche, quand bien même les femmes s'avèrent nettement sur-représentées dans l'échantillon et que, de l'aveu même de l'auteur, les individus qu'il a interrogés paraissent plutôt appartenir à des milieux favorisés. Cette omission est d'autant plus regrettable qu'elle semble résulter d'un choix opéré dès la conception de l'enquête, 
comme on le devine à la lecture de la note méthodologique ${ }^{2}$. Il reste donc à démontrer commet l'on peut «faire parler le DP du monde dans lequel nous vivons » (p. 6) en ignorant la place qu'y occupent ses adeptes. Quant à l'interprétation finale, elle nous semble fragilisée par le constat de l'appartenance sociale des enquêtés : si la lecture d'ouvrages de DP constitue une réaction à des injonctions auxquelles les individus manquent de ressources pour se conformer, comment expliquer qu'elle n'émane pas des milieux sociaux les moins dotés? L'ouvrage de Nicolas Marquis laisse ainsi un singulier goût d'inachevé, malgré ses incontestables qualités.

\footnotetext{
2 «Je n'[ai] pas posé de questions portant sur la "classe sociale" ou la "strate socioéconomique"» (p. 202).
} 